\title{
Flavonol Glycosides from Epimedium pubescens
}

\author{
Fengjuan Tu, ${ }^{a, \#}$ Yi DAI, ${ }^{b, \#}$ Zhihong YAO, ${ }^{b}$ Xinluan WANG, ${ }^{c, d}$ Xinsheng YAO, ${ }^{*}, a, b$ and Ling QIN ${ }^{c, d}$ \\ ${ }^{a}$ College of Traditional Chinese Materia Medica, Shenyang Pharmaceutical University; Shenyang 110016, P.R. China: \\ ${ }^{b}$ Institute of Traditional Chinese Medicine \& Natural Products, College of Pharmacy, Jinan University; Guangzhou \\ 510632, P. R. China: ' ${ }^{c}$ Musculoskeletal Research Laboratory, Department of Orthopaedics \& Traumatology, The Chinese \\ University of Hong Kong; Hong Kong, China: and ${ }^{d}$ Translational Medicine Research \& Development Center, Shenzhen \\ Institutes of Advanced Technology, Chinese Academy of Sciences; Shenzhen 518055, China.
}

Received May 5, 2011; accepted August 1,2011; published online August 25, 2011

Five new flavonol glycosides $(1,3,5-7)$ were isolated from the aerial parts of Epimedium pubescens MAXIM. along with two known compounds, sagittasine $C(2)$ and $4^{\prime}, 5$-dihydroxyl-8-(3,3-dimethylallyl)-flavonol 3- $O$-[ $\beta$-Dxylopyranosyl $(1 \rightarrow 3)-4-O$-acetyl- $\alpha$-L-rhamnopyranoside]-7- $O$ - $\beta$-D-glucopyranoside (4). The structures were elucidated on the basis of their 1D-, 2D-NMR, MS, UV and IR spectra data.

Key words Epimedium pubescens; Berberidaceae; flavonol glycoside

Epimedium pubescens MAXIM. (Berberidaceae), together with other four species E. brevicornum Maxim., E. sagittatum Maxim., E. wushanenes T. S. YING, and E. koreanum NAKAI, is used as the official source of Traditional Chinese Medicine (TCM) "Yinyanghuo" (Chinese Pharmacopoeia 2010 Edition). It is one of the most well known herbal medicines with tonic, anti-rheumatic and aphrodisiac effects. ${ }^{1,2}$ Previous chemical investigations on the geneus Epimedium have afforded a series of flavonol glycosides, ${ }^{3-7)}$ which possess multiple biological activities such as androgenic, ${ }^{8)}$ antioxidant, ${ }^{9}$ antiosteoporosis, ${ }^{10)}$ and antidepressant-like actions. ${ }^{11)}$ Our further chemical investigation on the aerial parts of E. pubescens MAXIM. resulted in the isolation of five new flavonol glycosides (compounds 1, 3, 5-7) and two known compounds, sagittasine $\mathrm{C}(\mathbf{2})^{3)}$ and $4^{\prime}, 5$-dihydroxy-8-(3,3dimethylallyl)-flavonol $3-O-[\beta$-D-xylopyranosyl $(1 \rightarrow 3)-4-O$ acetyl- $\alpha$-L-rhamnopyranoside]-7- $O-\beta$-D-glucopyranoside (4). ${ }^{12)}$ Their structures were elucidated on the basis of spectroscopic evidences (Fig. 1).

Compounds 1, 3, 5, $\mathbf{6}$ and $\mathbf{7}$ were obtained as yellow amorphous powder. The UV absorption bands of all the compounds were at about 205, 270, 320 and $350 \mathrm{~nm}$, suggesting the presence of flavonol skeleton in their structures.

The molecular formula of compound $\mathbf{1}$ was determined as $\mathrm{C}_{33} \mathrm{H}_{40} \mathrm{O}_{15}$ based on the high resolution-electrospray ionizationmass spectrum (HR-ESI-MS) $\left(m / z \quad 677.2448\left[\mathrm{C}_{33} \mathrm{H}_{41} \mathrm{O}_{15}\right]^{+}\right.$, Calcd for 677.2440). The ${ }^{1} \mathrm{H}-\mathrm{NMR}$ spectrum of 1 exhibited two singlet protons at $\delta 12.58(1 \mathrm{H}, \mathrm{s}, \mathrm{OH}-5)$ and $\delta 6.28(1 \mathrm{H}$, $\mathrm{s})$, respectively. Three coupled aromatic protons at $\delta 7.36$ $(1 \mathrm{H}, \mathrm{dd}, J=8.4,2.1 \mathrm{~Hz}), 7.34(1 \mathrm{H}, \mathrm{d}, J=2.1 \mathrm{~Hz})$, and 7.08 $(1 \mathrm{H}, \mathrm{d}, J=8.4 \mathrm{~Hz})$ suggested the presence of a 1,2,4-trisubsti- tuted benzene ring. A methoxy signal was obversed at $\delta 3.85$ $(3 \mathrm{H}, \mathrm{s})$. A serial of proton signals at $\delta 5.15(1 \mathrm{H}, \mathrm{t}, J=7.0 \mathrm{~Hz}$, H-12), 3.33 (2H, m, H-11), 1.68 (3H, s, H-15), 1.62 (3H, s, $\mathrm{H}-16)$ correlated with carbon signals at $\delta 122.4,25.4,17.7$, 21.2 in heteronuclear single quantum coherence (HSQC) spectrum respectively, suggesting the presence of a prenyl group. In addition, the proton signals at $\delta 5.34(1 \mathrm{H}, \mathrm{d}$, $\left.J=1.3 \mathrm{~Hz}, \mathrm{H}-1^{\prime \prime}\right) / 0.83\left(3 \mathrm{H}, \mathrm{d}, J=6.0 \mathrm{~Hz}, \mathrm{H}-6^{\prime \prime}\right)$, and $4.86(1 \mathrm{H}$, d, $\left.J=1.1 \mathrm{~Hz}, \mathrm{H}-1^{\prime \prime \prime}\right) / 1.08\left(3 \mathrm{H}, \mathrm{d}, J=6.2 \mathrm{~Hz}, \mathrm{H}-6^{\prime \prime \prime}\right)$ indicated the existence of two rhamnose moieties. It was further confirmed by the acid hydrolysis and HPLC analysis according to the method of Tanaka et al. ${ }^{13)}$ The absolute configuration of the sugars was determined to be the $L$ configuration. The methoxy group at $\delta 3.85$ was attached at the $\mathrm{C}-4^{\prime}(\delta$ 160.5) due to the characteristic heteronuclear multiple bond connectivity (HMBC) correlation. The aromatic carbon signals at $\delta$ 158.8, 98.5 and 103.7 were assigned to C-5, C-6 and C-10, due to their HMBC correlations with the hydroxy group at $\delta$ 12.58 (OH-5) (Fig. 2). Therefore, the carbon signals at $\delta$ 162.6 and 105.9 were then assigned to $\mathrm{C}-7$ and $\mathrm{C}-8$ according to their HMBC correlations with H-6 ( $\delta$ 6.28). The prenyl group was located at $\mathrm{C}-8$, which was supported by the HMBC correlation between $\mathrm{H}-11(\delta 3.33,2 \mathrm{H}, \mathrm{m})$ and $\mathrm{C}-8(\delta$ 105.9). Moreover, one rhamnose moiety was located at $\mathrm{C}-3$ of the aglycone according to the HMBC correlation of $\mathrm{H}$ $1 " / \mathrm{C}-3(\delta$ 134.2). And the other rhamnose moiety was located at $\mathrm{C}-2^{\prime \prime}$ of the inner one due to the HMBC correlations of $\mathrm{H}-1^{\prime \prime \prime} / \mathrm{C}-2^{\prime \prime}\left(\delta\right.$ 75.6) and $\mathrm{H}-2^{\prime \prime}(\delta 4.10,1 \mathrm{H}, \mathrm{m}) / \mathrm{C}-1^{\prime \prime \prime}(\delta$ 101.6). Thus, the structure of 1 was deduced as $4^{\prime}$-methoxyl3',5,7-trihydroxyl-8-(3,3-dimethylallyl)-flavonol 3-O- $\alpha$-Lrhamnopyranosyl $(1 \rightarrow 2)$ - $\alpha$-L-rhamnopyranoside.
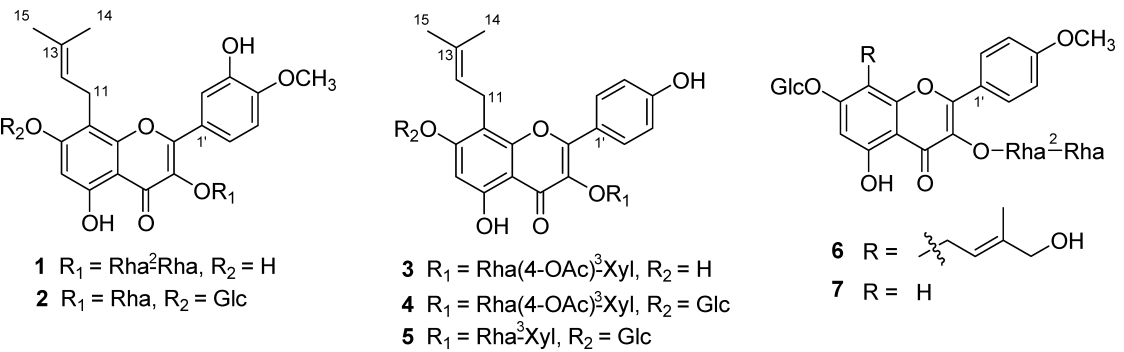

Fig. 1. Chemical Structures of $\mathbf{1}-\mathbf{7}$

* To whom correspondence should be addressed. e-mail: yaoxinsheng@vip.tom.com

\# These authors contributed equally to this work. 

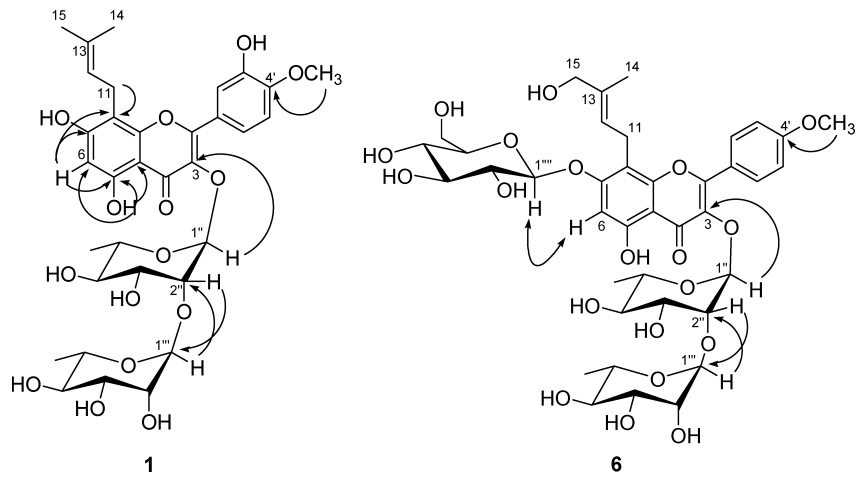

Fig. 2. Key HMBC $(\rightarrow)$ and ROESY $(\leftrightarrow)$ Correlations of $\mathbf{1}$ and $\mathbf{6}$

Compound 3 had the molecular formula $\mathrm{C}_{33} \mathrm{H}_{38} \mathrm{O}_{15}$ based on the HR-ESI-MS $\left(m / z 675.2296\left[\mathrm{C}_{33} \mathrm{H}_{29} \mathrm{O}_{15}\right]^{+}\right.$, Calcd for 675.2283). The ${ }^{1} \mathrm{H}-\mathrm{NMR}$ spectrum of 3 showed a singlet signal of OH-5 at $\delta 12.48(1 \mathrm{H}, \mathrm{s})$. A set of ortho-coupled doublet signals of four aromatic protons at $\delta 7.71,6.93$ (each $2 \mathrm{H}, \mathrm{d}, J=7.8 \mathrm{~Hz}$ ) and a one-proton singlet signal at $\delta 6.10$ $(1 \mathrm{H}, \mathrm{s})$ were also observed. The presence of an acetyl group was supported by a three-proton singlet signal at $\delta 1.97$ in the ${ }^{1} \mathrm{H}-\mathrm{NMR}$ spectrum and carbon signals at $\delta 169.7,20.8$ in the ${ }^{13} \mathrm{C}-\mathrm{NMR}$ spectrum. Proton signals at $\delta 5.17(1 \mathrm{H}, \mathrm{t}$, $J=5.9 \mathrm{~Hz}, \mathrm{H}-12), 3.34$ (2H, m. H-11) as well as two methyl groups signals at $\delta 1.67$ and 1.61 (each $3 \mathrm{H}, \mathrm{s}, \mathrm{H}-14,15)$ revealed the presence a prenyl group. It was further confirmed by the carbon signals at $\delta 21.3$ (C-11), 123.3 (C-12), 129.9 $(\mathrm{C}-13), 17.8(\mathrm{C}-14)$ and $25.4(\mathrm{C}-15)$ observed in the ${ }^{13} \mathrm{C}$ NMR spectrum. Additionally, two sugar moieties were observed with protons $\delta 5.30(1 \mathrm{H}$, br s $) / 0.71(3 \mathrm{H}, \mathrm{d}, J=6.2 \mathrm{~Hz})$ and $\delta 4.22(1 \mathrm{H}, \mathrm{d}, J=7.6 \mathrm{~Hz})$ in the ${ }^{1} \mathrm{H}-\mathrm{NMR}$ spectrum. They were identified as an L-rhamnose and a D-xylose by the acid hydrolysis experiment and HPLC analysis according to the method of Tanaka et al. ${ }^{13)}$ The aromatic methylene carbon signal at $\delta 99.5$ was assigned to C-6, due to its HMBC correlation with the hydroxy group at $\delta 12.48$ (OH-5). Therefore, the carbon signals at $\delta 158.9$ and 106.2 were then assigned to $\mathrm{C}-7$ and $\mathrm{C}-8$ according to their HMBC correlations with H-6 $(\delta 6.10)$. The location of the prenyl group at the $\mathrm{C}-8(\delta 106.2)$ position was supported by the chemical shift value of C-6 ( $\delta 99.5)$ in the ${ }^{13} \mathrm{C}-\mathrm{NMR}$ spectrum. $\left.{ }^{3,14-16}\right)$ It was further confirmed by the $\mathrm{HMBC}$ correlation between $\mathrm{H}-11(\delta 3.34)$ and $\mathrm{C}-8$. In the HMBC spectrum, a correlation between the H-1" $(\delta 5.30)$ and C-3 ( $\delta$ 134.2) were observed, indicating the rhamnose moiety was attached to $\mathrm{C}-3$ of the aglycone. The xylose moiety was located at C-3" ( $\delta 76.5)$ of the inner rhamnose moiety, according to the HMBC correlation of $\mathrm{H}-1^{\prime \prime \prime}(\delta 4.22) / \mathrm{C}-3^{\prime \prime}$. The acetyl group was located at $\mathrm{C}-4^{\prime \prime}$ of the rhamnose moiety due to the correlation between $\mathrm{H}-4^{\prime \prime}(\delta 4.85)$ and the carbonyl carbon of the acetyl group at $\delta$ 169.7. Therefore, the structure of 3 was elucidated to be 4',5,7-trihydroxyl-8-(3,3-dimethylallyl)-flavonol 3- $O$ - $\beta$-D-xylopyranosyl $(1 \rightarrow 3)-4-O$-acetyl- $\alpha$-L-rhamnopyranoside.

The molecular formula of Compound 5 was established as $\mathrm{C}_{37} \mathrm{H}_{46} \mathrm{O}_{19}$ by HR-ESI-MS $\left(m / z \quad 795.2726 \quad\left[\mathrm{C}_{37} \mathrm{H}_{47} \mathrm{O}_{19}\right]^{+}\right.$, Calcd for 795.2706). The NMR data of 5 were similar with those of 3, except for the loss of the acetyl group signals and additional signals of a glucose moiety. The anomeric proton at $\delta 5.00\left(1 \mathrm{H}, \mathrm{d}, J=7.3 \mathrm{~Hz}, \mathrm{H}-1^{\prime \prime \prime \prime}\right)$ indicated it to be $\beta$-glu- cose. The absolute configuration was further elucidated as D configuration by the acid hydrolysis experiment. ${ }^{13)}$ The glucose moiety was located at $\mathrm{C}-7$ of the aglycone due to the rotating frame Overhauser effect spectroscopy (ROESY) correlation of H-6 $(\delta 6.63) / \mathrm{H}-1^{\prime \prime \prime \prime}$. Thus, the structure of 5 was identified as 4',5-dihydroxyl-8-(3,3-dimethylallyl)-flavonol 3- $O$-[ $\beta$-D-xylopyranosyl $(1 \rightarrow 3)$ - $\alpha$-L-rhamnopyranozside $]-$ 7- $O$ - $\beta$-D-glucopyranoside.

Compound $\mathbf{6}$ was obtained with the molecular formula $\mathrm{C}_{39} \mathrm{H}_{50} \mathrm{O}_{20}$ by HR-ESI-MS $\left(m / z\right.$ 839.2982 $\left[\mathrm{C}_{39} \mathrm{H}_{51} \mathrm{O}_{20}\right]^{+}$, Calcd for 839.2968). The ${ }^{1} \mathrm{H}-\mathrm{NMR}$ spectrum of $\mathbf{6}$ suggested the presence of a chelated $5-\mathrm{OH}$ group $(\delta 12.61,1 \mathrm{H}, \mathrm{s})$, a penta-substituted benzene ring $(\delta 6.64,1 \mathrm{H}, \mathrm{s})$, a para-substituted benzene ring $(\delta 7.12,7.90$, each $2 \mathrm{H}, \mathrm{d}, J=2.1 \mathrm{~Hz})$, and a methoxy group $(\delta 3.85,3 \mathrm{H}, \mathrm{s})$. Proton signals at $\delta 5.37$ (1H, m, H-12), 3.73 (2H, br s, H-15), 3.60 (1H, m, H-11a), $3.47(1 \mathrm{H}, \mathrm{m}, \mathrm{H}-11 \mathrm{~b}), 1.66(3 \mathrm{H}, \mathrm{s}, \mathrm{H}-14)$ in the ${ }^{1} \mathrm{H}-\mathrm{NMR}$ spectrum, correlated with carbon signals at $\delta 120.9$ (C-12), 66.1 (C-15), 20.8 (C-11), 13.7 (C-14) in HSQC spectrum respectively, indicating the presence of a hydroxyprenyl group. Additionally, three anomeric protons were observed at $\delta 5.39$ $\left(1 \mathrm{H}, \mathrm{d}, J=1.5 \mathrm{~Hz}, \mathrm{H}-1^{\prime \prime}\right), 4.88\left(1 \mathrm{H}, \mathrm{d}, J=1.1 \mathrm{~Hz}, \mathrm{H}-1^{\prime \prime \prime}\right)$, and $5.01\left(1 \mathrm{H}, \mathrm{d}, J=7.5 \mathrm{~Hz}, \mathrm{H}-1^{\prime \prime \prime \prime}\right)$ respectively, suggesting the presence of three monosaccharide moieties. They were identified as two $\alpha$-L-rhamnose moieties and a $\beta$-D-glucose moiety by the acid hydrolysis experiment. ${ }^{13)}$ The methoxy group was located at $\mathrm{C}-4^{\prime}$ according to the HMBC correlation between the proton signal at $\delta 3.85$ and $C-4^{\prime}(\delta 161.4)$. One rhamnose moiety was located at $\mathrm{C}-3$ of the aglycone due to the HMBC correlation of $\mathrm{H}-1^{\prime \prime} / \mathrm{C}-3(\delta$ 134.5). The other rhamnose moiety was located at $\mathrm{C}-2^{\prime \prime}$ of the inner one due to the HMBC correlations of $\mathrm{H}-1^{\prime \prime \prime} / \mathrm{C}-2^{\prime \prime}(\delta 75.5)$ and $\mathrm{H}-2^{\prime \prime}(\delta$ $4.12,1 \mathrm{H}, \mathrm{m}) / \mathrm{C}-1^{\prime \prime \prime}(\delta$ 101.6). And the glucopyranose moiety was located at C-7 ( $\delta$ 160.6) according to the ROESY correlation between H-1'"' and H-6 ( $\delta$ 6.64) (Fig. 2). Therefore, compound 6 was elucidated as $4^{\prime}$-methoxyl-5-hydroxyl-8-(3methyl-4-hydroxyl-but-2-enyl)-flavonol 3-O-[ $\alpha$-L-rhamnopy$\operatorname{ranosyl}(1 \rightarrow 2)$ - $\alpha$-L-rhamnopyranoside]-7- $O$ - $\beta$-D-glucopyranoside.

Compound 7 was obtained with the molecular formula $\mathrm{C}_{34} \mathrm{H}_{42} \mathrm{O}_{19}$ by HR-ESI-MS $\left(m / z \quad 755.2402 \quad\left[\mathrm{C}_{34} \mathrm{H}_{43} \mathrm{O}_{19}\right]^{+}\right.$, Calcd for 755.2393). The NMR spectroscopic data of 7 were similar with those of $\mathbf{6}$, except for the loss of the signals of a hydroxyprenyl group. Thus, the structure of 7 was elucidated as $\quad 4^{\prime}$-methoxyl-5-hydroxyl-flavonol 3-O-[ $\alpha$-L-rhamnopyra$\operatorname{nosyl}(1 \rightarrow 2)-\alpha$-L-rhamnopyranoside]-7- $O$ - $\beta$-D-glucopyranoside.

\section{Experimental}

General Procedure UV spectra were recorded on a JASCO V-550 UV/Vis spectrometer. IR spectra were obtained using a JASCO FT/IR-480 plus spectrometer. ESI-MS spectra were recorded on a Finnigan LCQ Advantage MAX mass spectrometer. HR-ESI-MS spectra were acquired using Thermo-fishier LTQ Orbitrap XL mass spectrometer. 1D- and 2D-NMR spectra were measured with a Bruker AV-400 spectrometer. Open column chromatography was performed using silica gel (200-300 mesh, Qingdao Haiyang Chemical Group Corp., Qingdao, China), ODS $(50 \mu \mathrm{m}$, YMC, Japan), HW-40 (Tosoh, Japan) and Sephadex LH-20 (Pharmacia). Thin layer chromatography (TLC) was performed using percolated silica gel plates (silica gel $\mathrm{GF}_{254}, 1 \mathrm{~mm}$, Yantai). An agilent series $1200 \mathrm{HPLC}$ instrument equipped with a quaternary pump, a multiple wavelength detector, an auto sampler and a column compartment was used.

Plant Material The plant was supplied by Guizhou Tongjitang Pharmaceutical Co., Ltd., Guiyang, China, and identified by Professor Guang-Xiong 
Table 1. NMR Data of Aglycone Moiety for Compounds 1, 3, 5-7 (in DMSO- $d_{6}$ )

\begin{tabular}{|c|c|c|c|c|c|c|c|c|c|c|}
\hline \multirow{2}{*}{ No. } & \multicolumn{2}{|r|}{1} & \multicolumn{2}{|r|}{3} & \multicolumn{2}{|r|}{5} & \multicolumn{2}{|r|}{6} & \multicolumn{2}{|r|}{7} \\
\hline & $\delta_{\mathrm{C}}$ & $\delta_{\mathrm{H}}(J$ in $\mathrm{Hz})$ & $\delta_{\mathrm{C}}$ & $\delta_{\mathrm{H}}(J$ in $\mathrm{Hz})$ & $\delta_{\mathrm{C}}$ & $\delta_{\mathrm{H}}(J$ in $\mathrm{Hz})$ & $\delta_{\mathrm{C}}$ & $\delta_{\mathrm{H}}(J$ in $\mathrm{Hz})$ & $\delta_{\mathrm{C}}$ & $\delta_{\mathrm{H}}(J$ in $\mathrm{Hz})$ \\
\hline 2 & 156.6 & & 155.9 & & 157.7 & & 157.3 & & 157.4 & \\
\hline 3 & 134.2 & & 133.1 & & 134.0 & & 134.5 & & 134.8 & \\
\hline 4 & 177.7 & & 176.8 & & 178.1 & & 178.2 & & 177.9 & \\
\hline 5 & 158.8 & & 157.9 & & 159.0 & & 159.1 & & 160.9 & \\
\hline 6 & 98.5 & $6.28 \mathrm{~s}$ & 99.5 & $6.10 \mathrm{~s}$ & 98.1 & $6.63 \mathrm{~s}$ & 98.1 & $6.64 \mathrm{~s}$ & 99.4 & $6.48 \mathrm{~d}(2.1)$ \\
\hline 7 & 162.6 & & 158.9 & & 160.4 & & 160.6 & & 163.1 & \\
\hline 8 & 105.9 & & 106.2 & & 108.3 & & 108.1 & & 94.7 & $6.78 \mathrm{~d}(2.1)$ \\
\hline 9 & 153.7 & & 153.9 & & 152.9 & & 153.0 & & 156.1 & \\
\hline 10 & 103.7 & & 102.0 & & 105.5 & & 105.1 & & 105.8 & \\
\hline 11 & 21.2 & $3.38 \mathrm{~m}$ & 21.3 & $3.34 \mathrm{~m}$ & 21.4 & $\begin{array}{l}3.34 \mathrm{~m} \\
3.55 \mathrm{~m}\end{array}$ & 20.8 & $\begin{array}{l}3.47 \mathrm{~m} \\
3.60 \mathrm{~m}\end{array}$ & & \\
\hline 12 & 122.4 & $5.15 \mathrm{t}(7.0)$ & 123.3 & 5.17 t (5.9) & 122.2 & $5.16 \mathrm{t}(6.9)$ & 120.9 & $5.37 \mathrm{~m}$ & & \\
\hline 13 & 130.9 & & 129.9 & & 131.0 & & 135.6 & & & \\
\hline 14 & 17.7 & $1.68 \mathrm{~s}$ & 17.8 & $1.67 \mathrm{~s}$ & 17.8 & $1.69 \mathrm{~s}$ & 13.7 & $1.66 \mathrm{~s}$ & & \\
\hline 15 & 25.4 & $1.62 \mathrm{~s}$ & 25.4 & $1.61 \mathrm{~s}$ & 25.4 & $1.60 \mathrm{~s}$ & 66.1 & $3.73 \mathrm{br} \mathrm{s}$ & & \\
\hline $1^{\prime}$ & 122.5 & & 120.6 & & 120.3 & & 122.1 & & 121.9 & \\
\hline $2^{\prime}$ & 115.4 & $7.34 \mathrm{~d}(2.1)$ & 130.2 & $7.71 \mathrm{~d}(8.7)$ & 130.7 & $7.80 \mathrm{~d}(8.7)$ & 130.6 & $7.90 \mathrm{~d}(9.0)$ & 130.6 & $7.88 \mathrm{~d}(8.9)$ \\
\hline $3^{\prime}$ & 146.4 & & 115.3 & $6.93 \mathrm{~d}(8.7)$ & 115.4 & $6.94 \mathrm{~d}(8.7)$ & 114.0 & $7.12 \mathrm{~d}(9.0)$ & 114.1 & $7.12 \mathrm{~d}(8.9)$ \\
\hline $4^{\prime}$ & 150.0 & & 160.3 & & 160.4 & & 161.4 & & 161.4 & \\
\hline $5^{\prime}$ & 111.7 & $7.08 \mathrm{~d}(8.4)$ & 115.3 & $6.93 \mathrm{~d}(8.7)$ & 115.4 & $6.94 \mathrm{~d}(8.7)$ & 114.0 & $7.12 \mathrm{~d}(9.0)$ & 114.1 & $7.12 \mathrm{~d}(8.9)$ \\
\hline $6^{\prime}$ & 120.6 & $7.36 \mathrm{dd}(8.4,2.1)$ & 130.2 & $7.71 \mathrm{~d}(8.7)$ & 130.7 & $7.80 \mathrm{~d}(8.7)$ & 130.6 & $7.90 \mathrm{~d}(9.0)$ & 130.6 & $7.88 \mathrm{~d}(8.9)$ \\
\hline $4^{\prime}-\mathrm{OCH}_{3}$ & 55.7 & $3.85 \mathrm{~s}$ & & & & & 55.5 & $3.85 \mathrm{~s}$ & 55.5 & $3.85 \mathrm{~s}$ \\
\hline $5-\mathrm{OH}$ & & $12.58 \mathrm{~s}$ & & $12.48 \mathrm{~s}$ & & $12.57 \mathrm{~s}$ & & $12.61 \mathrm{~s}$ & & $12.65 \mathrm{~s}$ \\
\hline
\end{tabular}

Zhou, College of Pharmacy, Jinan University. A voucher specimen was deposited in the Institute of Traditional Chinese Medicine and Natural Products, Jinan University, Guangzhou, China.

Extraction and Isolation The aerial parts of E. pubescens MAXIM $(2 \mathrm{~kg}$ ) were extracted twice with $60 \%$ ethanol. After removal of the ethanol in vacuo, the extract ( $245 \mathrm{~g}$ ) was chromatographied over Diaion HP-20 resin, eluted with water, $30 \%$ and $95 \%$ ethanol in successive. The $95 \%$ ethanol eluate $(80 \mathrm{~g})$ was then subjected to a silica-gel column chromatography (CC) eluted with chloroform-methanol in gradient to give fourteen fractions. Fraction $7\left(\mathrm{CHCl}_{3}-\mathrm{MeOH}\right.$ 9:1 eluent) was then subjected to ODS CC eluted with $\mathrm{MeOH}-\mathrm{H}_{2} \mathrm{O}$ in gradient. And nine subfractions were obtained $(7 \mathrm{~A}-\mathrm{I})$. The subfraction $7 \mathrm{H}$ (eluted with $60 \% \mathrm{MeOH}-\mathrm{H}_{2} \mathrm{O}$ ) was further separated by Sephadex LH-20 eluted with $\mathrm{CHCl}_{3}-\mathrm{MeOH}(1: 1)$. Compound $3(16 \mathrm{mg})$ was obtained after the purification by HW-40 CC eluted with $50 \%$ $\mathrm{MeOH}-\mathrm{H}_{2} \mathrm{O}$. Fraction $11\left(\mathrm{CHCl}_{3}-\mathrm{MeOH}\right.$ 8:2 eluent) was chromatographied on ODS CC with $\mathrm{MeOH}-\mathrm{H}_{2} \mathrm{O}$ in gradient to yield 7 subfractions (11A to $\mathrm{G}$ ). The subfraction $11 \mathrm{~B}$ (eluted with $20 \% \mathrm{MeOH}-\mathrm{H}_{2} \mathrm{O}$ ) was applied to repeated Sephadex $\mathrm{LH}-20 \mathrm{CC}$ eluted with $\mathrm{CHCl}_{3}-\mathrm{MeOH}(1: 1)$ and 35\% $\mathrm{MeOH}-\mathrm{H}_{2} \mathrm{O}$, respectively. Then the eluate was separated by preparative HPLC with $50 \% \mathrm{MeOH}-\mathrm{H}_{2} \mathrm{O}$ to yield compounds $6(21 \mathrm{mg})$ and $7(10 \mathrm{mg})$. Fraction $10\left(\mathrm{CHCl}_{3}-\mathrm{MeOH} 8: 2\right.$ eluent) was also subjected to ODS CC eluted with $\mathrm{MeOH}-\mathrm{H}_{2} \mathrm{O}$ in gradient to yield 9 subfractions $(10 \mathrm{~A}-\mathrm{I})$. Fraction $10 \mathrm{~F}$ (eluted with $50 \% \mathrm{MeOH}-\mathrm{H}_{2} \mathrm{O}$ ) was further subjected to Sephadex LH-20 eluted with $55 \% \mathrm{MeOH}-\mathrm{H}_{2} \mathrm{O}$ and then separated by preparative HPLC (Ultimate $^{\mathrm{TM}}$ XB-C18, $5 \mu \mathrm{m}, 21.2 \times 250 \mathrm{~mm}$, Welch) with $50 \%$ $\mathrm{MeOH}-\mathrm{H}_{2} \mathrm{O}$ to yield compounds $2(8 \mathrm{mg})$ and $5(26 \mathrm{mg})$. Fraction $10 \mathrm{G}$ (eluted with $50 \% \mathrm{MeOH}-\mathrm{H}_{2} \mathrm{O}$ ) was purified by Sephadex $\mathrm{LH}-20$ with $60 \%$ $\mathrm{MeOH}-\mathrm{H}_{2} \mathrm{O}$ to yield compound $4(68 \mathrm{mg}$ ). Fraction 10I (eluted with $50 \%$ $\mathrm{MeOH}-\mathrm{H}_{2} \mathrm{O}$ ) was subjected to Sephadex LH-20 eluted with $60 \%$ $\mathrm{MeOH}-\mathrm{H}_{2} \mathrm{O}$, and then separated by preparative HPLC (Ultimate ${ }^{\mathrm{TM}} \mathrm{XB}-\mathrm{C} 18$, $5 \mu \mathrm{m}, 21.2 \times 250 \mathrm{~mm}$, Welch) with $65 \% \mathrm{MeOH}-\mathrm{H}_{2} \mathrm{O}$ to yield compound $\mathbf{1}$ (9 mg).

Acid Hydrolysis and HPLC Analysis The absolute configuration of the sugar moieties in the structures were determined by the method of Tanaka et al. ${ }^{13)}$ Compound 1 ( $2 \mathrm{mg}$ ) was hydrolyzed with $2 \mathrm{M} \mathrm{HCl}$ for $2 \mathrm{~h}$ at $90{ }^{\circ} \mathrm{C}$. The mixture was evaporated to dryness under a vacuum, and then the residue was dissolved in $\mathrm{H}_{2} \mathrm{O}$ and extracted with $\mathrm{CHCl}_{3}$. The aqueous layer was collected. After drying in vacuo, the residue was dissolved in pyridine ( $1 \mathrm{ml})$ containing L-cysteine methyl ester $(1 \mathrm{mg})$ (Sigma, U.S.A.) and heated at $60{ }^{\circ} \mathrm{C}$ for $1 \mathrm{~h}$. Then, $o$-tolyl isothiocyanate $(5 \mu \mathrm{l})$ (Alfa Aesar, U.K.) was added to the mixture, which was heated at $60^{\circ} \mathrm{C}$ for $1 \mathrm{~h}$. The reaction mixture was directly analyzed by reversed-phase HPLC. Analytical HPLC was performed on a Cosmosil $5 \mathrm{C}_{18}$-MS-II column $(250 \times 4.6 \mathrm{~mm}$ i.d., $5 \mu \mathrm{m}$,
Nacalai Tesque Inc., Japan) at $35^{\circ} \mathrm{C}$ with isocratic elution of $25 \% \mathrm{CH}_{3} \mathrm{CN}$ containing $0.1 \%$ formic acid for $40 \mathrm{~min}$ and subsequent washing of the column with $90 \% \mathrm{CH}_{3} \mathrm{CN}$ at a flow rate $0.8 \mathrm{ml} / \mathrm{min}$. Peaks were detected by a $\mathrm{UV}$ detector at $250 \mathrm{~nm}$. One peak of the derivatives of 1 was obversed at $t_{\mathrm{R}}$ 29.1 (L-Rha) min. The mixture of standard monosaccharides, such as Lrhamnose, D-glucose, L-glucose, D-xylose, and L-xylose (Sigma, U.S.A.), were subjected to the same method. The peaks of the standard monosaccharide derivatives were recorded at $t_{\mathrm{R}} 15.9$ (L-Glc), 17.2 (D-Glc), 18.7 (L-Xyl), 20.0 (D-Xyl), and 29.2 (L-Rha) min. Following the above procedure, the derivatives of 2,6 and 7 gave two peaks at $t_{\mathrm{R}} 17.2-17.3$ (D-Glc) and 29.229.3 (L-Rha) min, respectively. The derivatives of 3 gave two peaks at $t_{\mathrm{R}} 20.1$ (D-Xyl) and 29.3 (L-Rha) min. Those of 4 and 5 both gave three peaks at $t_{\mathrm{R}}$ 17.2-17.3 (D-Glc), 20.0-20.1 (D-Xyl), and 29.1-29.2 (L-Rha) min.

4'-Methoxyl-3',5,7-trihydroxyl-8-(3,3-dimethylallyl)-flavonol 3- $O-\alpha$-LRhamnopyranosyl $(1 \rightarrow 2)$ - $\alpha$-L-rhamnopyranoside (1): Yellow powder; UV $\lambda_{\text {max }}(\mathrm{MeOH}) \mathrm{nm}(\log \varepsilon): 205$ (4.47), 258 (4.13, sh), 270 (4.17), 346 (3.91); IR (KBr) cm ${ }^{-1}: 3402,2933,1653,1509,1046 ;{ }^{1} \mathrm{H}-$ and ${ }^{13} \mathrm{C}-\mathrm{NMR}$ data (see Tables 1, 2); ESI-MS (positive) $m / z: 699[\mathrm{M}+\mathrm{Na}]^{+}, 1375[2 \mathrm{M}+\mathrm{Na}]^{+}, 677$ $[\mathrm{M}+\mathrm{H}]^{+}$, ESI-MS (negative) $m / z: 675[\mathrm{M}-\mathrm{H}]^{-}, 1351[2 \mathrm{M}-\mathrm{H}]^{-}$; HR-ESIMS $m / z$ : $677.2448[\mathrm{M}+\mathrm{H}]^{+}\left(\right.$Calcd for $\left.\mathrm{C}_{33} \mathrm{H}_{41} \mathrm{O}_{15}, 677.2440\right)$.

Sagittasine C (2): Yellow powder; UV $\lambda_{\max }(\mathrm{MeOH}) \mathrm{nm}(\log \varepsilon): 205$ (4.50), 258 (4.14, sh), 269 (4.15), 346 (3.91); IR (KBr) cm ${ }^{-1}: 3388,2925$, 1651, 1599, 1076; ${ }^{1} \mathrm{H}-\mathrm{NMR}$ (DMSO- $\left.d_{6}, 400 \mathrm{MHz}\right) \delta: 12.59$ (1H, s, 5-OH), $7.40\left(1 \mathrm{H}, \mathrm{dd}, J=8.5,2.1 \mathrm{~Hz}, \mathrm{H}-6^{\prime}\right), 7.38\left(1 \mathrm{H}, \mathrm{d}, J=2.1 \mathrm{~Hz}, \mathrm{H}-2^{\prime}\right), 7.09(1 \mathrm{H}$, d, $\left.J=8.5 \mathrm{~Hz}, \mathrm{H}-5^{\prime}\right), 6.62(1 \mathrm{H}, \mathrm{s}, \mathrm{H}-6), 5.25\left(1 \mathrm{H}, \mathrm{d}, J=1.3 \mathrm{~Hz}, \mathrm{H}-1^{\prime \prime}\right), 5.17$ $(1 \mathrm{H}, \mathrm{t}, J=7.0 \mathrm{~Hz}, \mathrm{H}-12), 5.00\left(1 \mathrm{H}, \mathrm{d}, J=7.5 \mathrm{~Hz}, \mathrm{H}-1^{\prime \prime \prime}\right), 4.00(1 \mathrm{H}$, br s, H-2"), $3.86\left(3 \mathrm{H}, \mathrm{s}, 4^{\prime}-\mathrm{OCH}_{3}\right), 3.72\left(1 \mathrm{H}, \mathrm{m}, \mathrm{H}-6^{\prime \prime \prime \prime} \mathrm{a}\right), 3.54$ (1H, m, H-11a), 3.53 (1H, m, H-3"), 3.48 (1H, m, H-6"'"b), 3.43 (2H, m, H-11b, H-5"'), 3.35-3.28 (overlapped in HDO, H-2"', $3^{\prime \prime \prime}$ ), 3.26-3.12 (3H, m, H-4"', H-4", H-5"), 1.70 $(3 \mathrm{H}, \mathrm{s}, \mathrm{H}-14), 1.61(3 \mathrm{H}, \mathrm{s}, \mathrm{H}-15), 0.81\left(3 \mathrm{H}, \mathrm{d}, J=6.0 \mathrm{~Hz}, \mathrm{H}-6{ }^{\prime \prime}\right) ;{ }^{13} \mathrm{C}-\mathrm{NMR}$ data (DMSO- $\left.d_{6}, 100 \mathrm{MHz}\right) \delta$ : 178.3 (C-4), 160.5 (C-7), 159.0 (C-5), 157.4 (C-2), 152.9 (C-9), 150.2 (C-4'), 146.4 (C-3'), 134.6 (C-3), 131.1 (C-13), $122.4\left(\mathrm{C}-1^{\prime}\right), 122.1$ (C-12), $120.8\left(\mathrm{C}-6^{\prime}\right), 115.5\left(\mathrm{C}-2^{\prime}\right), 111.7\left(\mathrm{C}-5^{\prime}\right), 108.3$ (C-8), 105.5 (C-10), 102.0 (C-1"), 100.6 (C-1"'), 98.1 (C-6), 77.2 (C-5"'), $76.6\left(\mathrm{C}-3^{\prime \prime \prime}\right), 73.3\left(\mathrm{C}-2^{\prime \prime \prime}\right), 71.2\left(\mathrm{C}-4^{\prime \prime}\right), 70.6\left(\mathrm{C}-5^{\prime \prime}\right), 70.3\left(\mathrm{C}-3^{\prime \prime}\right), 70.0\left(\mathrm{C}-2^{\prime \prime}\right)$, 69.7 (C-4"'), 60.6 (C-6"'), $55.7\left(4^{\prime}-\mathrm{OCH}_{3}\right), 25.4$ (C-15), 21.4 (C-11), 17.7 (C-14), 17.4 (C-6"); ESI-MS (positive) $m / z$ : $715[\mathrm{M}+\mathrm{Na}]^{+}, 1407$ $[2 \mathrm{M}+\mathrm{Na}]^{+}, 693[\mathrm{M}+\mathrm{H}]^{+}, \mathrm{ESI}-\mathrm{MS}$ (negative) $m / z: 691[\mathrm{M}-\mathrm{H}]^{-}, 1383$ $[2 \mathrm{M}-\mathrm{H}]^{-}$; HR-ESI-MS $m / z: 693.2404[\mathrm{M}+\mathrm{H}]^{+}\left(\right.$Calcd for $\mathrm{C}_{33} \mathrm{H}_{41} \mathrm{O}_{16}$, 693.2389).

4',5,7-Trihydroxyl-8-(3,3-dimethylallyl)-flavonol 3-O- $\beta$-D-Xylopyranosyl$(1 \rightarrow 3)-4-O$-acetyl- $\alpha$-L-rhamnopyranoside (3): Yellow powder; UV $\lambda_{\max }$ $(\mathrm{MeOH}) \mathrm{nm}(\log \varepsilon): 204$ (4.43, sh), 270 (4.25), 316 (3.97, sh), 350 (3.91); 
Table 2. NMR Data of Sugar Moieties for Compounds 1, 3, 5-7 (in DMSO- $d_{6}$ )

\begin{tabular}{|c|c|c|c|c|c|c|c|c|c|c|}
\hline \multirow{2}{*}{ No. } & \multicolumn{2}{|r|}{1} & \multicolumn{2}{|r|}{3} & \multicolumn{2}{|r|}{5} & \multicolumn{2}{|r|}{6} & \multicolumn{2}{|r|}{7} \\
\hline & $\delta_{\mathrm{C}}$ & $\delta_{\mathrm{H}}(J$ in $\mathrm{Hz})$ & $\delta_{\mathrm{C}}$ & $\delta_{\mathrm{H}}(J$ in $\mathrm{Hz})$ & $\delta_{\mathrm{C}}$ & $\delta_{\mathrm{H}}(J$ in $\mathrm{Hz})$ & $\delta_{\mathrm{C}}$ & $\delta_{\mathrm{H}}(J$ in $\mathrm{Hz})$ & $\delta_{\mathrm{C}}$ & $\delta_{\mathrm{H}}(J$ in $\mathrm{Hz})$ \\
\hline \multicolumn{11}{|l|}{ Rhamnose-1 } \\
\hline $1^{\prime \prime}$ & 100.7 & $5.34 \mathrm{~d}(1.3)$ & 101.2 & 5.30 br s & 101.8 & 5.30 br s & 100.6 & $5.41 \mathrm{~d}(1.3)$ & 100.7 & $5.39 \mathrm{~d}(1.5)$ \\
\hline $2^{\prime \prime}$ & 75.6 & $4.10 \mathrm{~m}$ & 69.6 & 4.12 br s & 69.5 & 4.13 br s & 75.5 & $4.11 \mathrm{~m}$ & 75.5 & $4.12 \mathrm{~m}$ \\
\hline $3^{\prime \prime}$ & 70.1 & $3.63 \mathrm{~m}$ & 76.5 & $3.80 \mathrm{~m}$ & 80.9 & $3.56 \mathrm{~m}$ & 70.1 & $3.59 \mathrm{~m}$ & 70.1 & $3.61 \mathrm{~m}$ \\
\hline $4^{\prime \prime}$ & 71.4 & $3.26-3.10 \mathrm{o}$ & 71.4 & $4.84 \mathrm{t}(10.0)$ & 69.9 & $3.40-3.27 \mathrm{o}$ & 71.3 & $3.23-3.07 \mathrm{o}$ & 71.3 & $3.22-3.08 \mathrm{o}$ \\
\hline $5^{\prime \prime}$ & 70.4 & $3.26-3.10 \mathrm{o}$ & 68.2 & $3.32 \mathrm{~m}$ & 70.5 & $3.27-3.05 \mathrm{o}$ & 70.4 & $3.23-3.07 \mathrm{o}$ & 70.4 & $3.22-3.08 \mathrm{o}$ \\
\hline $6^{\prime \prime}$ & 17.5 & $0.83 \mathrm{~d}(6.0)$ & 17.0 & $0.71 \mathrm{~d}(6.2)$ & 17.4 & $0.84 \mathrm{~d}(6.1)$ & 17.5 & $0.81 \mathrm{~d}(5.6)$ & 17.4 & $0.81 \mathrm{~d}(5.6)$ \\
\hline \multirow{2}{*}{$4^{\prime \prime}-\mathrm{O}-\mathrm{COCH}_{3}$} & & & 169.7 & & & & & & & \\
\hline & & & 20.8 & $1.97 \mathrm{~s}$ & & & & & & \\
\hline \multicolumn{11}{|l|}{ Rhamnose-2 } \\
\hline $1^{\prime \prime \prime}$ & 101.6 & $4.86 \mathrm{~d}(1.1)$ & & & & & 101.6 & 4.88 br s & 101.6 & $4.88 \mathrm{~d}(1.1)$ \\
\hline $2^{\prime \prime \prime}$ & 70.2 & $3.68 \mathrm{~m}$ & & & & & 70.2 & $3.68 \mathrm{~m}$ & 70.2 & $3.68 \mathrm{~m}$ \\
\hline $3^{\prime \prime \prime}$ & 70.6 & $3.50-3.31 \mathrm{o}$ & & & & & 70.7 & $3.36 \mathrm{~m}$ & 70.7 & $3.36 \mathrm{~m}$ \\
\hline $4^{\prime \prime \prime}$ & 71.9 & $3.26-3.10 \mathrm{o}$ & & & & & 71.9 & $3.23-3.07 \mathrm{o}$ & 71.9 & $3.22-3.08 \mathrm{o}$ \\
\hline $5^{\prime \prime \prime}$ & 68.7 & $3.50-3.31 \mathrm{o}$ & & & & & 68.8 & $3.39 \mathrm{~m}$ & 68.8 & $3.39 \mathrm{~m}$ \\
\hline $6^{\prime \prime \prime}$ & 17.5 & $1.08 \mathrm{~d}(6.2)$ & & & & & 17.6 & $1.11 \mathrm{~d}(6.2)$ & 17.6 & $1.10 \mathrm{~d}(6.1)$ \\
\hline \multicolumn{11}{|l|}{ Xylose } \\
\hline $1^{\prime \prime \prime}$ & & & 105.5 & $4.22 \mathrm{~d}(7.6)$ & 105.8 & $4.32 \mathrm{~d}(7.3)$ & & & & \\
\hline $2^{\prime \prime \prime}$ & & & 72.9 & $2.98 \mathrm{~m}$ & 73.8 & $3.08 \mathrm{~m}$ & & & & \\
\hline $3^{\prime \prime \prime}$ & & & 76.7 & $3.11 \mathrm{~m}$ & 76.2 & $3.14 \mathrm{~m}$ & & & & \\
\hline $4^{\prime \prime \prime}$ & & & 69.5 & $3.30 \mathrm{~m}$ & 69.5 & $3.40-3.27 \mathrm{o}$ & & & & \\
\hline \multirow{2}{*}{$5^{\prime \prime \prime}$} & & & 65.7 & $3.77 \mathrm{~m}$ & 65.7 & $3.75 \mathrm{~m}$ & & & & \\
\hline & & & & $3.14 \mathrm{~m}$ & & $3.12 \mathrm{~m}$ & & & & \\
\hline \multicolumn{11}{|l|}{ Glucose } \\
\hline $1^{\prime \prime \prime \prime}$ & & & & & 100.5 & $5.07 \mathrm{~d}(7.3)$ & 99.9 & $5.01 \mathrm{~d}(7.5)$ & 100.6 & $5.07 \mathrm{~d}(7.3)$ \\
\hline $2^{\prime \prime \prime \prime}$ & & & & & 73.3 & $3.30 \mathrm{~m}$ & 73.1 & $3.27 \mathrm{~m}$ & 73.3 & $3.30 \mathrm{~m}$ \\
\hline $3^{\prime \prime \prime \prime}$ & & & & & 76.6 & $3.30 \mathrm{~m}$ & 76.4 & $3.30 \mathrm{~m}$ & 76.6 & $3.31 \mathrm{~m}$ \\
\hline $4^{\prime \prime \prime \prime}$ & & & & & 69.6 & $3.27-3.05 \mathrm{o}$ & 69.5 & $3.23-3.07 \mathrm{o}$ & 69.6 & $3.22-3.08 \mathrm{o}$ \\
\hline $5^{\prime \prime \prime \prime}$ & & & & & 77.2 & $3.43 \mathrm{~m}$ & 77.2 & $3.44 \mathrm{~m}$ & 77.1 & $3.43 \mathrm{~m}$ \\
\hline \multirow[t]{2}{*}{$6^{\prime \prime \prime \prime}$} & & & & & 60.6 & $3.71 \mathrm{~m}$ & 60.6 & $3.72 \mathrm{~m}$ & 60.6 & $3.72 \mathrm{~m}$ \\
\hline & & & & & & $3.48 \mathrm{~m}$ & & $3.48 \mathrm{~m}$ & & $3.48 \mathrm{~m}$ \\
\hline
\end{tabular}

"o" refers to peaks overlapped with other signals.

IR $(\mathrm{KBr}) \mathrm{cm}^{-1}: 3419,2926,1651,1612,1044 ;{ }^{1} \mathrm{H}$ - and ${ }^{13} \mathrm{C}-\mathrm{NMR}$ data (see Tables 1, 2); ESI-MS (positive) $m / z: 697[\mathrm{M}+\mathrm{Na}]^{+}, 1371[2 \mathrm{M}+\mathrm{Na}]^{+}$, ESIMS (negative) $m / z: 673[\mathrm{M}-\mathrm{H}]^{-}$; HR-ESI-MS m/z: $675.2296[\mathrm{M}+\mathrm{H}]^{+}$ (Calcd for $\mathrm{C}_{33} \mathrm{H}_{39} \mathrm{O}_{15}, 675.2283$ ).

4',5-Dihydroxyl-8-(3,3-dimethylallyl)-flavonol 3- $O$-[ $\beta$-D-Xylopyranosyl$(1 \rightarrow 3)-4-O$-acetyl- $\alpha$-L-rhamnopyranoside]-7- $O$ - $\beta$-D-glucopyranoside (4): Yellow powder; UV $\lambda_{\max }(\mathrm{MeOH}) \mathrm{nm}(\log \varepsilon): 204(4.59$, sh), 270 (4.34), 318 (4.08, sh), 348 (4.03); IR (KBr) cm ${ }^{-1}: 3420,2926,1651,1601,1074 ;{ }^{1} \mathrm{H}-$ NMR (DMSO- $\left.d_{6}, 400 \mathrm{MHz}\right) \delta: 12.53(1 \mathrm{H}, \mathrm{s}, 5-\mathrm{OH}), 7.78(2 \mathrm{H}, \mathrm{d}, J=8.8 \mathrm{~Hz}$, H-2', 6' $), 6.96\left(2 \mathrm{H}, \mathrm{d}, J=8.8 \mathrm{~Hz}, \mathrm{H}-3^{\prime}, 5^{\prime}\right), 6.63(1 \mathrm{H}, \mathrm{s}, \mathrm{H}-6), 5.32(1 \mathrm{H}, \mathrm{br} \mathrm{s}$, H-1"), 5.17 (1H, t, $J=7.0 \mathrm{~Hz}, \mathrm{H}-12), 5.00$ (1H, d, $\left.J=7.4 \mathrm{~Hz}, \mathrm{H}-1^{\prime \prime \prime \prime}\right), 4.85$ $\left(1 \mathrm{H}, \mathrm{t}, J=10.0 \mathrm{~Hz}, \mathrm{H}-4^{\prime \prime}\right), 4.22\left(1 \mathrm{H}, \mathrm{d}, J=7.6 \mathrm{~Hz}, \mathrm{H}-1^{\prime \prime \prime}\right), 4.14(1 \mathrm{H}$, br s, H$\left.2^{\prime \prime}\right), 3.80\left(1 \mathrm{H}, \mathrm{dd}, J=10.1,2.3 \mathrm{~Hz}, \mathrm{H}-3^{\prime \prime}\right), 3.77$ (1H, m, H-5"'a), $3.73(1 \mathrm{H}, \mathrm{m}$, H-6"'"'a), 3.57 (1H, m, H-11a), 3.48 (1H, m, H-6"'”b), 3.44 (1H, m, H-11b), $3.43\left(1 \mathrm{H}, \mathrm{m}, \mathrm{H}-5^{\prime \prime \prime \prime}\right), 3.40-3.25$ (overlapped in HDO, H-4"', H-5", H-2'"', H$\left.3^{\prime \prime \prime \prime}\right), 3.17$ (1H, m, H-4"'"'), 3.14 (1H, m, H-5"'b), 3.08 (1H, m, H-3"'), 2.98 (1H, m, H-2"' ), 1.97 (3H, s, 4"-OAc), 1.68 (3H, s, H-14), 1.60 (3H, s, H-15), $0.72\left(3 \mathrm{H}, \mathrm{d}, J=6.2 \mathrm{~Hz}, \mathrm{H}-6^{\prime \prime}\right) ;{ }^{13} \mathrm{C}-\mathrm{NMR}$ data (DMSO-d $\left.6,100 \mathrm{MHz}\right) \delta: 178.1$ (C-4), 169.7 (4"-O- $\left.\mathrm{COCH}_{3}\right), 160.5$ (C-7), 160.5 (C-4'), 159.0 (C-5), 157.7 (C-2), 153.0 (C-9), 133.8 (C-3), 131.0 (C-13), 130.6 (C-2', 6'), 122.1 (C12), $120.3\left(\mathrm{C}-1^{\prime}\right), 115.4$ (C-3', 5'), 108.3 (C-8), 105.6 (C-1"'), 105.5 (C-10), $101.3\left(\mathrm{C}-1^{\prime \prime}\right), 100.6\left(\mathrm{C}-1^{\prime \prime \prime \prime}\right), 98.1$ (C-6), $77.2\left(\mathrm{C}-5^{\prime \prime \prime \prime}\right), 76.7\left(\mathrm{C}-3^{\prime \prime \prime}\right), 76.6(\mathrm{C}-$ $\left.3^{\prime \prime \prime \prime}\right), 76.5\left(\mathrm{C}-3^{\prime \prime}\right), 73.3\left(\mathrm{C}-2^{\prime \prime \prime \prime}\right), 72.9\left(\mathrm{C}-2^{\prime \prime \prime}\right), 71.3\left(\mathrm{C}-4^{\prime \prime}\right), 69.6\left(\mathrm{C}-4^{\prime \prime \prime \prime}\right), 69.6$ $\left(\mathrm{C}-2^{\prime \prime}\right), 69.5\left(\mathrm{C}-4^{\prime \prime \prime}\right), 68.4\left(\mathrm{C}-5^{\prime \prime}\right), 65.8$ (C-5"'), 60.6 (C-6"'"'), 25.4 (C-15), 21.4 (C-11), 20.8 (4"-O-COCH${ }_{3}$ ), 17.8 (C-14), 17.0 (C-6"); ESI-MS (positive) $m / z: 859[\mathrm{M}+\mathrm{Na}]^{+}, 1695[2 \mathrm{M}+\mathrm{Na}]^{+}, 837[\mathrm{M}+\mathrm{H}]^{+}$, ESI-MS (negative) $m / z: 835[\mathrm{M}-\mathrm{H}]^{-}$; HR-ESI-MS $m / z: 837.2825[\mathrm{M}+\mathrm{H}]^{+}$(Calcd for $\left.\mathrm{C}_{39} \mathrm{H}_{49} \mathrm{O}_{20}, 837.2812\right)$.

4',5-Dihydroxyl-8-(3,3-dimethylallyl)-flavonol 3- $O$-[ $\beta$-D-Xylopyranosyl$(1 \rightarrow 3)-\alpha$-L-rhamnopyranoside]-7- $O-\beta$-D-glucopyranoside $(5)$ : Yellow powder; UV $\lambda_{\text {max }}(\mathrm{MeOH}) \mathrm{nm}(\log \varepsilon): 204(4.38, \mathrm{sh}), 270$ (4.13), $321(3.87, \mathrm{sh})$, 350 (3.85); IR (KBr) cm ${ }^{-1}: 3409,2924,1651,1600,1075 ;{ }^{1} \mathrm{H}-$ and ${ }^{13} \mathrm{C}-$ NMR data (see Tables 1, 2); ESI-MS (positive) $m / z: 817[\mathrm{M}+\mathrm{Na}]^{+}, 1611$ $[2 \mathrm{M}+\mathrm{Na}]^{+}, 795[\mathrm{M}+\mathrm{H}]^{+}$, ESI-MS (negative) $m / z: 793[\mathrm{M}-\mathrm{H}]^{-}, 1587$
$[2 \mathrm{M}-\mathrm{H}]^{-}$; HR-ESI-MS m/z: $795.2726[\mathrm{M}+\mathrm{H}]^{+}$(Calcd for $\mathrm{C}_{37} \mathrm{H}_{47} \mathrm{O}_{19}$ 795.2706).

4'-Methoxyl-5-hydroxyl-8-(3-methyl-4-hydroxyl-but-2-enyl)-flavonol 3$O$-[ $\alpha$-L-Rhamnopyranosyl $(1 \rightarrow 2)$ - $\alpha$-L-rhamnopyranoside]-7- $O$ - $\beta$-D-glucopyranoside (6): Yellow powder; UV $\lambda_{\max }(\mathrm{MeOH}) \mathrm{nm}(\log \varepsilon): 204$ (4.62, sh), 270 (4.38), 316 (4.14, sh), 348 (4.07); IR (KBr) $\mathrm{cm}^{-1}: 3408,2930,1651$, 1597, 1062; ${ }^{1} \mathrm{H}$ - and ${ }^{13} \mathrm{C}-\mathrm{NMR}$ data (see Tables 1,2 ); ESI-MS (positive) $\mathrm{m} / \mathrm{z}$ : $861[\mathrm{M}+\mathrm{Na}]^{+}, 839[\mathrm{M}+\mathrm{H}]^{+}$, ESI-MS (negative) $m / z: 873[\mathrm{M}+\mathrm{Cl}]^{-}$; HRESI-MS $m / z$ : $839.2982[\mathrm{M}+\mathrm{H}]^{+}\left(\right.$Calcd for $\left.\mathrm{C}_{39} \mathrm{H}_{51} \mathrm{O}_{20}, 839.2968\right)$.

4 '-Methoxyl-5-hydroxyl-flavonol 3- $O$-[ $\alpha$-L-Rhamnopyranosyl $(1 \rightarrow 2)-\alpha$-Lrhamnopyranoside]-7- $O$ - $\beta$-D-glucopyranoside (7): Yellow powder; UV $\lambda_{\max }$ $(\mathrm{MeOH}) \mathrm{nm}(\log \varepsilon): 204$ (4.41, sh), 266 (4.17), 317 (3.97, sh), 342 (3.98); IR (KBr) $\mathrm{cm}^{-}:$3420, 2931, 1654, 1603, 1066; ${ }^{1} \mathrm{H}-$ and ${ }^{13} \mathrm{C}-\mathrm{NMR}$ data (see Tables 1, 2); ESI-MS (positive) $m / z: 777[\mathrm{M}+\mathrm{Na}]^{+}, 755[\mathrm{M}+\mathrm{H}]^{+}$, ESI-MS (negative) $m / z: 789[\mathrm{M}+\mathrm{Cl}]^{-}, 1507[2 \mathrm{M}-\mathrm{H}]^{-}$; HR-ESI-MS $m / z: 755.2402$ $[\mathrm{M}+\mathrm{H}]^{+}\left(\right.$Calcd for $\left.\mathrm{C}_{34} \mathrm{H}_{43} \mathrm{O}_{19}, 755.2393\right)$.

Acknowledgements We thank Wen $\mathrm{Xu}$ (Laboratory of Chinese Drugs Pharmaceutics, Guangdong Hospital of Traditional Chinese Medicine, Guangzhou, China) for measuring the HR-ESI-MS data. We also thank Zhen-qiang Mu and Ming-yan Liu for measuring the NMR and ESI-MS data. And we are grateful to Guizhou Tongjitang Pharmaceutical Co., Ltd. for providing the plant material. This work was supported by Grants from the National Natural Science Foundation of China (NSFC-RGC$30831160510)$.

\section{References}

1) Zhao H. Y., Sun J. H., Fan M. X., Fan L., Zhou L., Han J., Wang B. R., Guo D. A., J. Chromatogr. A, 119, 157-181 (2008).

2) Zhao H. Y., Fan L., Zhou L., Han J., Wang B. R., Guo D. A., Helv. Chim. Acta, 90, 2186-2195 (2007).

3) Wang G. J., Tsai T. H., Lin L. C., Phytochemistry, 68, 2455-2464 (2007).

4) Uriburu M. L., Gil R. R., Sosa V. E., de la Fuente J. R., Phytochemistry, 
68, 1295-1299 (2007).

5) Yu C. Y., Song L. N., Chen G., Chin. Chem. Lett., 20, 842-844 (2009).

6) Eun S. K., Mi K. K., Hyun K. K., Young I. P., Mi S. D., Nat. Prod. Sci., 14, 233-238 (2008).

7) Luo G. J., Ci X. X., Ren R., Wu Z. Y., Li H. M., Li H. Z., Li R. T., Deng X. M., Planta Med., 75, 843-847 (2009).

8) Yang J. Y., Yu Q. H., Wu J., J. Shenyang Pharm. Univ., 15, 98-101 (1998).

9) Wang T., Zhang J. C., Chen Y., Huang F., Yang M., Xiao P. G., China J. Chin. Mat. Med., 32, 715-718 (2007).

10) Peng S. L., Zhang G., He Y. X., Wang X. L., Leung P. C., Leung K., Qin L., Bone, 45, 534-544 (2009).
11) Pan Y., Kong L. D., Xia X., Zhang W. Y., Xia Z. H., Jiang F. X., Pharmacol. Biochem. Behav., 82, 686-694 (2005).

12) Ueda T., Nakajima K., Chin M., Mihashi H., JP Patent 04159295 (1992).

13) Tanaka T., Nakashima T., Ueda T., Tomii K., Kouno I., Chem. Pharm. Bull., 55, 899-901 (2007).

14) Li W. K., Zhang R. Y., Xiao P. G., Phytochemistry, 43, 527-530 (1996).

15) Carvalho M. G., Suzart L. R., Cavatti L. C., Kaplan M. A. C., J. Braz. Chem. Soc., 19, 1423-1428 (2008).

16) Zhang P. C., Wang S., Wu Y., Chen R. Y., Yu D. Q., J. Nat. Prod., 64, 1206-1209 (2001). 